\title{
TRANSIENT POPULATION DYNAMICS IN PERIODIC MATRIX MODELS: METHODOLOGY AND EFFECTS OF CYCLIC PERMUTATIONS
}

\author{
Shana K. Mertens, ${ }^{1,4}$ Jonathan M. Yearsley, ${ }^{2}$ Frank van den Bosch, ${ }^{1}$ and Christopher A. Gilligan ${ }^{3}$ \\ ${ }^{1}$ Rothamsted Research, Harpenden AL5 2JQ UK \\ ${ }^{2}$ Université de Lausanne, Départment d'écology et d'évolution, Biophore, CH-1015, Lausanne Switzerland \\ ${ }^{3}$ Department of Plant Sciences, University of Cambridge, Downing Street, Cambridge CB2 3EA UK
}

\begin{abstract}
Many biological populations are subject to periodically changing environments such as years with or without fire, or rotation of crop types. The dynamics and management options for such populations are frequently investigated using periodic matrix models. However the analysis is usually limited to long-term results (asymptotic population growth rate and its sensitivity to perturbations of vital rates). In non-periodic matrix models it has been shown that long-term results may be misleading as populations are rarely in their stable structure. We therefore develop methods to analyze transient dynamics of periodic matrix models. In particular, we show how to calculate the effects of perturbations on population size within and at the end of environmental cycles. Using a model of a weed population subject to a crop rotation, we show that different cyclic permutations produce different patterns of sensitivity of population size and different population sizes. By examining how the starting environment interacts with the initial conditions, we explain how different patterns arise. Such understanding is critical to developing effective management and monitoring strategies for populations subject to periodically recurring environments.
\end{abstract}

Key words: adaptive management; elasticity; periodic matrix model; Polygonum persicaria; population dynamics; population management; sensitivity; transient dynamics.

\section{INTRODUCTION}

Many biological populations are subject to periodically recurring environments, which have different impacts on vital rates, e.g., survival may be higher in one environment than another. Examples of populations subject to periodic environments are plants exposed to seasons with and without fire (Silva et al. 1991, Hoffman 1999, Satterthwaite et al. 2002), cycles of flood and drought (Beissinger 1995), exploited organisms subject to periodic harvesting (Escalante et al. 2004, Endress et al. 2004), and weeds growing in crop rotations (Mertens et al. 2002). Populations subject to periodic environments are often modeled using a simple extension of a nonseasonal matrix population model (Caswell and Trevisan 1994, Caswell 2001).

Asymptotic analysis provides useful insights into the dynamics of populations (Benton and Grant 1999). However, asymptotic results can be misleading in formulating management decisions because conditions are unlikely to remain stable for long enough for the long-term dynamics to be reached (Fox and Gurevitch 2000, Yearsley 2004, Koons et al. 2005). Consequently, analysis shortly after a perturbation is made and a focus on population size may be more appropriate for management purposes and is essential in adaptive management

Manuscript received 19 October 2005; revised 28 February 2006; accepted 6 March 2006. Corresponding Editor: A. M. de Roos.

${ }^{4}$ E-mail: shana.mertens@bbsrc.ac.uk programs (Walters 1986, Shea et al. 2002). Analysis of transient dynamics is also useful in testing models when time scales do not allow experiments to continue to the point that asymptotic dynamics might be reached.

The transient dynamics of matrix population models can be investigated in a variety of ways, for example by examining the damping ratio (Lefkovitch 1971, Caswell 2001) or through calculating population momentum (Keyfitz 1971). It is only recently, though, that analytic methods have been developed for calculating transient sensitivities of population size and the short-term growth rate (Fox and Gurevitch 2000, Yearsley 2004). While it is possible to use a simulation approach, it becomes very time consuming because of the infinite number of combinations of changes that can be made (Fox and Gurevitch 2000). More importantly, the analytic results can be used to gain a deeper understanding of the system. For example, bounds can be placed on the range of sensitivities over the set of all initial conditions. The methods developed by Fox and Gurevitch (2000) and Yearsley (2004), however, apply only to populations exposed to constant (noncyclic) environments.

\section{Periodic systems and permutation of environments}

Periodic environments can be described as either independent or dependent. When an environment is dependent on a previous environment, it is not possible to alter its position in the cycle relative to a previous environment, for example, the temperate seasons. In contrast, the order of environments can be changed 
when they are independent of each other and therefore can be used as a management tool. There are many economically and environmentally important examples of systems composed wholly or partly of independent environments such as rotations of crops or pesticides (Liebman and Dyck 1993, May et al. 2005), periodic imposition of anti-trampling measures to protect an endangered plant (Gross et al. 1998), or periodic harvesting strategies (Endress et al. 2004).

Due to the fact that matrix multiplication is not commutative (see, e.g., Strang 1980), a key implication of asymptotic analysis of periodic matrix models is that different noncyclic permutations of a system of independent environments will lead to different asymptotic population growth rates (for examples see Caswell [2001]). Cyclic permutations, though, will have identical asymptotic growth rates and patterns of sensitivities of the growth rate because the patterns of environments are identical, and just out of phase (e.g., for two environments, $\mathrm{C}$ and $\mathrm{W}$, the sequence $\mathrm{CWCWCW}$ produces the same long-term population dynamics as WCWCWC). However, because different stable population structures are associated with each environment, one might expect that different cyclic permutations will lead to different population sizes and different short-term sensitivities of population size due to the interaction with the initial population structure.

\section{Periodic systems and dynamics within the environmental cycle}

Regardless of whether a system is composed of dependent or independent environments, another question is how the timing of observations affects the sensitivity of population size to perturbations of vital rates. For example, in a population exposed to the temperate seasons, one might expect that the effects of perturbations to fecundity will be different depending on whether they are observed after the spring or after the winter. This requires having a method to calculate the effects of perturbations on population size within the environmental cycle. Asymptotic methods, however, are focused on the effects of perturbations on the population growth rate over the entire environmental cycle (Caswell and Trevisan 1994, Caswell 2001).

\section{Aims of this study}

In this paper, we extend the transient demographic analyses of Fox and Gurevitch (2000) and Yearsley (2004) to the analysis of periodic matrix models, and in particular we develop a method to examine the effects of perturbations at any time within an environmental cycle. Using the new methods, we investigate how cyclic permutations and initial conditions affect population size in the long term and its sensitivity to perturbations in the short term for a weed Polygonum persicaria subject to a periodically changing set of crops (crop rotation). In Appendix A, we carry out the same analysis for a model of the tropical savanna grass Andropogon semiberbis (Silva et al. 1991), whose vital rates are a function of the plant's exposure to fire. Finally, we discuss the implications of the results for the observation and management of populations subject to periodically changing environments, and provide some general insights that have implications for the interpretation of non-periodic and periodic sensitivity analysis.

\section{Methods: Calculating Transient Effects of Perturbations \\ Periodic matrix models}

The dynamics of a structured population subject to periodically changing environments have frequently been simulated using matrix population models (Silva et al. 1991, Caswell and Trevisan 1994, Caswell 2001, Lesnoff 1999). In a periodic environment, a population is assumed to repeatedly experience the same sequence of $N$ environments. This sequence of environments is called a cycle and provides a natural time scale for the system (one cycle being one time step). Using a matrix population model approach, the evolution of population size in a periodic environment is then described by

$$
\mathbf{n}(t)=\mathbf{A}^{t} \mathbf{n}(0)
$$

where $\mathbf{n}(0)$ is the vector of initial population sizes, $\mathbf{n}(t)$ is the population vector after $t$ cycles and $\mathbf{A}$ is the population transition matrix for one complete cycle (Caswell and Trevisan 1994, Caswell 2001).

Each environment can be expressed as a population transition matrix whose elements give the demographic parameters of the population in that environment. The transition matrix A combines the transition matrices for these individual environments in a way which depends upon the number and the order of the environments. Specifically, the matrix $\mathbf{A}$ can be written as

$$
\mathbf{A}=\mathbf{P}^{(N)} \mathbf{P}^{(N-1)} \ldots \mathbf{P}^{(2)} \mathbf{P}^{(1)}
$$

where $\mathbf{P}^{(h)}$ is the population transition matrix for the $h$ th environmental phase in the cycle. As a particular environment can occur more than once in a cycle, it is useful to refer to environments as "phases" in a particular cycle. For example, in the cycle WCCWC, the environment $\mathrm{W}$ occurs in the first and fourth phases. A complete summary of the notation used in this paper is given in Table 1.

\section{Transient sensitivity}

Methods for calculating various transient sensitivities of a non-periodic matrix model have recently been developed (Fox and Gurevitch 2000, Yearsley 2004). The transient sensitivity of the population size to changes in the element $a_{i j}$ of the matrix $\mathbf{A}$ is

$$
\frac{\partial \mathbf{n}(t)}{\partial a_{i j}}=\underbrace{\mathbf{S}\left(t ; a_{i j}\right) \frac{n(0)}{\left\|\mathbf{w}^{(1)}\right\|}}_{\text {Independent of } \mathbf{n}(0)}+\underbrace{\Delta \mathbf{S}\left(t ; a_{i j}\right) \Delta \mathbf{n}}_{\text {Linearly dependent on } \mathbf{n}(0)}
$$

where $\mathbf{n}(t)$ is the vector of population size at time $t, \mathbf{w}^{(1)}$ is the dominant right eigenvector of $\mathbf{A}$ and 
TABle 1. List of symbols and definitions used in this paper.

\begin{tabular}{ll}
\hline \hline \multicolumn{1}{c}{ Symbol } & \multicolumn{1}{c}{ Definition } \\
\hline$N$ & Number of environmental phases. \\
$h$ & A particular environmental phase that is perturbed. \\
$g$ & A particular environmental phase after which the effect of a perturbation is observed. \\
$\mathbf{A}$ & The transition matrix for one entire cycle. \\
$a_{i j}$ & The element in the $i$ th row and $j$ th column of $\mathbf{A}$. \\
$\mathbf{P}^{(h)}$ & The transition matrix for the $h$ th environmental phase. \\
$p_{k l}^{(h)}$ & The element in the $k$ th row and $l$ th column of $\mathbf{P}^{(h)}$. \\
$\lambda_{i}$ & The $i$ th eigenvalue associated with $\mathbf{A}$. \\
$\mathbf{w}^{(i)}$ & The right eigenvector associated with $\lambda_{i}$. \\
$\mathbf{n}(0)$ & The initial population vector. \\
$\mathbf{n}(t)$ & The population vector after the $i$ th cycle. \\
$n_{m}(t)$ & The $m$ th element of $\mathbf{n}(\mathbf{t})$. \\
$n(t)$ & The total population size after the $t$ th cycle. \\
$\mathbf{n}(t ; h)$ & The population vector after the $h$ th phase of the $t$ th cycle. \\
$\mathbf{n}(t ; g)$ & The population vector after the $g$ th phase of the $i$ th cycle. \\
$x$ & The complex conjugate of the scalar $x$. \\
$\mathbf{x}^{*}$ & The complex conjugate transpose of vector $\mathbf{x}$. \\
$\|\mathbf{x}\|=\sum_{i}\left|x_{i}\right|$ & The absolute sum of the elements of vector $\mathbf{x}$ (the L1-norm of $\mathbf{x})$. \\
$\langle\mathbf{x}, \mathbf{y}\rangle=\sum_{i} \mathrm{x}_{\mathrm{i}} \mathrm{y}_{\mathrm{i}}$ & The scalar product of vectors $\mathbf{x}$ and $\mathbf{y}$. \\
$x y \equiv \mathbf{x} \otimes \mathbf{y}^{*}$ & The vector direct product of vectors $\mathbf{x}$ and $\mathbf{y}$ giving a matrix $M_{i j}=x_{i} \bar{y}_{j}$. \\
\hline
\end{tabular}

$$
\Delta \mathbf{n}=\mathbf{n}(0)-\frac{n(0)}{\left\|\mathbf{w}^{(1)}\right\|} \mathbf{w}^{(1)}
$$

is the deviation of the initial population vector away from $\mathbf{w}^{(1)}$. The vector $\mathbf{S}\left(t ; a_{i j}\right)$ and the matrix $\Delta \mathbf{S}\left(t ; a_{i j}\right)$ can be calculated from $\mathbf{A}$ :

$$
\begin{aligned}
& \mathbf{S}\left(t ; a_{i j}\right)=t \lambda_{1}^{t-1} \frac{\partial \lambda_{1}}{\partial a_{i j}} \mathbf{w}^{(1)}+\sum_{m>1} \frac{\lambda_{1}^{t}-\lambda_{m}^{t}}{\lambda_{1}-\lambda_{m}} \bar{v}_{i}^{(m)} w_{j}^{(1)} \mathbf{w}^{(m)} \\
& \Delta \mathbf{S}\left(t ; a_{i j}\right)= \sum_{m} t \lambda_{m}^{t-1} \frac{\partial \lambda_{m}}{\partial a_{i j}} \mathbf{w}^{(m)} \otimes \mathbf{v}^{(m)_{*}} \\
&+\sum_{n \neq m} \frac{\lambda_{m}^{t}-\lambda_{n}^{t}}{\lambda_{m}-\lambda_{n}} \bar{v}_{i}^{(m)} w_{j}^{(n)} w^{(m)} \otimes \mathbf{v}^{(m)_{*}} .
\end{aligned}
$$

The vector $\mathbf{S}\left(t ; a_{i j}\right)$ quantifies the contribution that the stable stage structure makes to the transient sensitivity, while the matrix $\Delta \mathbf{S}\left(t ; a_{i j}\right)$ quantifies the contribution that the initial deviations from the stable stage distribution make toward the transient sensitivity (see Yearsley [2004] for a derivation of these results). Eqs. 5 and 6 require that no two eigenvalues of the transition matrix, $\mathbf{A}$, are the same. The equations can be modified to allow for repeated eigenvalues by following the approach used for eigenvector-eigenvalue relations (Elhay et al. 1999). If the eigenvalues of $\mathbf{A}$ are to be calculated accurately the matrix must not be ill conditioned (Press et al. 1996).

For a periodic system, a change in an element of the transition matrix A bears no simple relationship to environmental changes, so a standard analysis using $\mathbf{A}$ is difficult to interpret. A more useful analysis of a periodic system would quantify the effect of a change in one environmental phase (i.e., an element, $p_{k l}^{(h)}$, in one of the matrices $\mathbf{P}^{(h)}$ ). This can be achieved by extending the transient demographic analysis of Eq. 3 following many of the same techniques used by Caswell and Trevisan
(1994). These techniques relate a change in the $p_{k l}^{(h)}$ element of the $\mathbf{P}^{(h)}$ phase to changes in $\mathbf{A}$. Formally, this is achieved by using the chain rule:

$$
\frac{\partial \mathbf{n}(t)}{\partial p_{k l}^{(h)}}=\sum_{i, j} \frac{\partial \mathbf{n}(t)}{\partial a_{i j}} \frac{\partial a_{i j}}{\partial p_{k l}^{(h)}}
$$

where

$$
\frac{\partial a_{i j}}{\partial p_{k l}^{(h)}}=d_{i k}^{(+h)} d_{l j}^{(-h)}
$$

and $d_{i k}^{(+h)}$ and $d_{l j}^{(-h)}$ are elements, respectively, of the matrices

$$
\begin{aligned}
& \mathbf{D}^{(+h)} \equiv \mathbf{P}^{(N)} \cdots \mathbf{P}^{(h+1)} \\
& \mathbf{D}^{(-h)} \equiv \mathbf{P}^{(h-1)} \cdots \mathbf{P}^{(1)}
\end{aligned}
$$

and $\mathbf{D}^{(+N)}=\mathbf{D}^{(-1)}=\mathbf{I}$ are the identity matrices. Eq. 8 arises because the matrix $\mathbf{A}$ can be written as $\mathbf{D}^{(+h)} \mathbf{P}^{(h)} \mathbf{D}^{(-h)}$. Consequently, taking the derivative of $\mathbf{A}$ with respect to an element of $\mathbf{P}^{(h)}$ will lead to a matrix product containing the matrix $d\left(\mathbf{P}^{(h)}\right) / d p_{k l}$, whose elements are zero, except for the $k l$ th element, which is one. The method underlying Eqs. 8-10 is the same as that used by Lesnoff et al. (2003) in developing a more efficient method to calculate asymptotic sensitivities of periodic matrix models.

The transient sensitivity for a periodic system to changes in $p_{k l}^{(h)}$ can now be calculated by substituting Eq. 3 and Eq. 8 into Eq. 7. Working through the algebra, the expressions for the transient sensitivity and expressions for $\mathbf{S}(t)$ and $\Delta \mathbf{S}(t)$ can be written with respect to perturbations to an element $p_{k l}^{(h)}$ :

$$
\frac{\partial \mathbf{n}(t)}{\partial p_{k l}^{(h)}}=\mathbf{S}\left[t ; p_{k l}^{(h)}\right] \frac{n(0)}{\left\|\mathbf{w}^{(1)}\right\|}+\Delta \mathbf{S}\left[t ; p_{k l}^{(h)}\right] \Delta \mathbf{n}
$$




$$
\begin{aligned}
\mathbf{S}\left[t ; p_{k l}^{(h)}\right] & =\sum_{i, j} d_{i k}^{(+h)} d_{l j}^{(-h)} \mathbf{S}\left(t ; a_{i j}\right) \\
\Delta \mathbf{S}\left[t ; p_{k l}^{(h)}\right] & =\sum_{i, j} d_{i k}^{(+h)} d_{l j}^{(-h)} \Delta \mathbf{S}\left(t ; a_{i j}\right) .
\end{aligned}
$$

All the analytic methods for non-periodic environments (Fox and Gurevitch 2000, Yearsley 2004) can now be applied to periodic environments. In particular, the elasticities of the population size can be calculated (Fox and Gurevitch 2000):

$$
\mathbf{e}_{k l}^{(h)}(t)=p_{k l}^{(h)} \mathbf{N}(t)^{-1} \frac{\partial \mathbf{n}(t)}{\partial p_{k l}^{(h)}}
$$

where the matrix $\mathbf{N}(t)^{-1}$ is the inverse of $\operatorname{diag}(\mathbf{n}(t))$. The transient elasticities give the proportional effect on population size of a proportional change in a demographic parameter, $p_{k l}^{(h)}$ (de Kroon et al. 2000, Caswell 2001). Analogous to the non-periodic case, the sum of the elasticities of each phase is always equal to $t$ :

$$
\sum_{k, l} \mathbf{e}_{k l}^{(h)}(t)=t
$$

Transient sensitivities and elasticities can be scaled by time (Fox and Gurevitch 2000), or in the periodic case, by the number of cycles $t$. In the long term, the timescaled elasticities of population size are the same as the asymptotic elasticities of population growth rate.

The Supplement contains the Matlab scripts for carrying out the calculations described above and for calculating the immediate sensitivities and elasticities described in the next section.

\section{Within-cycle sensitivity}

The transient sensitivity of the population size at the end of each cycle (Eqs. 11-13) is not very informative about the sensitivity of the population part way through a cycle. Often the sensitivity within the environmental cycle is of interest as it allows more flexibility in observing the effects of perturbations. Essentially, this involves either back or forward projecting the end-ofcycle sensitivity by the product of the environmental matrices occurring, respectively, after or up-to the point of observation:

$$
\frac{\partial \mathbf{n}(t ; g)}{\partial p_{k l}^{(h)}}=\left[\mathbf{D}^{(+g)}\right]^{-1} \frac{\partial \mathbf{n}(t)}{\partial p_{k l}^{(h)}} \quad \text { if } g \geq h
$$

and

$$
\frac{\partial \mathbf{n}(t ; g)}{\partial p_{k l}^{(h)}}=\mathbf{D}^{(-[g+1])} \frac{\partial \mathbf{n}(t-1)}{\partial p_{k l}^{(h)}} \quad \text { if } g<h
$$

where $h$ is the phase being perturbed, $g$ is the phase where the sensitivity is observed and $\left[\mathbf{D}^{(+g)}\right]^{-1}$ is the inverse of $\mathbf{D}^{(+g)}$. $\mathbf{D}^{(+g)}$ and $\mathbf{D}^{(-[g+1])}$ are given by Eqs. 9 and 10. Appendix B contains the derivation of Eqs. 16a and $b$.
For example, if the cycle is the four temperate seasons and the population is projected from autumn to autumn, then the sequence is fall-winter-spring-summer and the annual projection matrix is given by $\mathbf{A}=\mathbf{U}^{(4)} \mathbf{S}^{(3)} \mathbf{W}^{(2)} \mathbf{F}^{(1)}$. To observe the effect perturbations being made in autumn, on the population after winter, Eq. 16a is evaluated with $h=1$ and $g=2$ so that $\mathbf{D}^{(+2)}=\mathbf{U}^{(4)} \mathbf{S}^{(3)}$. Eq. $16 \mathrm{~b}$ would be applied, say, when the effects are observed after winter $(g=2)$, but perturbations occur in spring $(h=3)$, giving $\mathbf{D}^{(-3)}=\mathbf{W}^{(2)} \mathbf{F}^{(1)}$.

A particularly useful application of Eq. 16a lies in comparing the sensitivities associated with perturbations to the same environmental phase but in different cyclic permutations. A natural way to compare sensitivities between cyclic permutations is to evaluate the sensitivities immediately after the environmental phase being perturbed, e.g., when $g=h$ in Eq. 16a. We call such sensitivities "immediate sensitivities."

Following a similar argument as for the end-of-cycle elasticities, the within-cycle elasticities can be calculated as

$$
\begin{aligned}
& \mathbf{e}_{k l}^{(h)}(t ; h ; g) \\
& =p_{k l}^{(h)}\left\{\mathbf{D}^{(+g)} \operatorname{diag}[\mathbf{n}(t ; g)]\right\}^{-1} \frac{\partial \mathbf{n}(t)}{\partial p_{k l}^{(h)}} \quad \text { if } g \geq h
\end{aligned}
$$

and

$$
\begin{aligned}
& \mathbf{e}_{k l}^{(h)}(t ; h ; g) \\
& =p_{k l}^{(h)}\{\operatorname{diag}[\mathbf{n}(t ; g)]\}^{-1} \mathbf{D}^{(-[g+1])} \frac{\partial \mathbf{n}(t-1)}{\partial p_{k l}^{(h)}} \quad \text { if } g<h
\end{aligned}
$$

where $\mathbf{n}(t ; g)$ is the population vector after the $g$ th phase of the $t$ th cycle. Analogous to the immediate sensitivities, the immediate elasticities are calculated by evaluating Eq. 17a when $g=h$.

\section{Sensitivity of total population size}

Eq. 11 contains a lot of information that can be difficult to interpret. One simplification is to concentrate upon the total population size, $n(t)$, rather than the population vector, $\mathbf{n}(t)$. The transient sensitivity of the total population size is simply the sum of the sensitivities of the population vector:

$$
\frac{\partial n(t)}{\partial p_{k l}^{(h)}}=\sum_{m} \frac{\partial n_{m}(t)}{\partial p_{k l}^{(h)}}=S_{\|}\left[t ; p_{k l}^{(h)}\right] \frac{n(0)}{\left\|\mathbf{w}^{(1)}\right\|}+\left\langle\Delta \mathbf{S}_{\|}\left[t ; p_{k l}^{(h)}\right], \Delta \mathbf{n}\right\rangle
$$

where the scalar $S_{\|}\left(t ; p_{k l}^{(h)}\right)$ and the row vector $\Delta \mathbf{S}_{\|}(t$; $\left.p_{k l}^{(h)}\right)$ are the sum of the elements in each column of $\mathbf{S}\left(t ; p_{k l}^{(h)}\right)$ and $\Delta \mathbf{S}\left(t ; p_{k l}^{(h)}\right)$, respectively. The result of Eq. 18 is a simple number, and the decomposition involves nothing more complicated than vectors (i.e., $\Delta \mathbf{S}_{\|}\left(t ; p_{k l}^{(h)}\right)$ and $\Delta \mathbf{n})$. However, Eq. 18 is still a function of time, and depends upon the initial population vector. 
TABle 2. Parameter values for the model of $P$. persicaria.

\begin{tabular}{|c|c|c|c|c|c|c|c|c|c|}
\hline \multirow[b]{3}{*}{ Crop and depth class } & \multicolumn{9}{|c|}{ Parameter } \\
\hline & \multirow[b]{2}{*}{$\alpha_{k}$} & \multirow[b]{2}{*}{$\mu_{k}$} & \multirow[b]{2}{*}{$v_{l}$} & \multirow[b]{2}{*}{$\gamma_{l}$} & \multirow[b]{2}{*}{$\sigma_{l}$} & \multicolumn{4}{|c|}{$\delta_{k l}$} \\
\hline & & & & & & 1 & 2 & 3 & 4 \\
\hline \multicolumn{10}{|l|}{ Carrots } \\
\hline 1 & 0.45 & 1.0 & 0.001 & 400 & 1 & 0.70 & 0.33 & 0.02 & 0.00 \\
\hline 2 & 0.0 & 0.3 & 0.001 & 400 & 1 & 0.23 & 0.50 & 0.15 & 0.00 \\
\hline 3 & 0.0 & 0.2 & 0.001 & 400 & 1 & 0.06 & 0.15 & 0.68 & 0.16 \\
\hline 4 & 0.0 & 0.2 & 0.001 & 400 & 1 & 0.02 & 0.01 & 0.16 & 0.84 \\
\hline \multicolumn{10}{|l|}{ Wheat } \\
\hline 1 & 0.15 & 0.2 & 0.6 & 90 & 1 & 0.02 & 0.21 & 0.37 & 0.29 \\
\hline 2 & 0.0 & 0.2 & 0.6 & 90 & 1 & 0.11 & 0.27 & 0.26 & 0.10 \\
\hline 3 & 0.0 & 0.2 & 0.6 & 90 & 1 & 0.40 & 0.30 & 0.20 & 0.12 \\
\hline 4 & 0.0 & 0.2 & 0.6 & 90 & 1 & 0.46 & 0.21 & 0.18 & 0.48 \\
\hline
\end{tabular}

Notes: All parameter values are from Mertens et al. (2002), except those for seed movement, which are from Cousens and Moss (1990). The symbols are defined as $\alpha_{l}$, seedling emergence from each depth class; $v_{l}$, survival of plants emerged from depth class $l ; \gamma_{l}$, seed production of plants emerged from depth class $l ; \delta_{k l}$, movement of seeds from class $l$ to class $k ; \mu_{l}$, mortality of seeds at each soil depth before plowing; and $\sigma_{k}$, survival of seeds at each soil depth after plowing. The shallowest soil depth is class 1 , and the deepest is class 4 .

Using Eq. 18, the elasticity of total population size can also be calculated using

$$
e_{k l}^{(h)}=\frac{p_{k l}^{(h)}}{n(t)} \frac{\partial n(t)}{\partial p_{k l}^{(h)}} .
$$

Similarly, the within-cycle sensitivities and elasticities of the total population size can also be calculated.

\section{RESULTS \\ Example}

In order to examine how cyclic permutations affect transient population dynamics, we use a model of a weed population subject to a crop rotation (a set of periodically changing crops). The model is that based on Mertens et al. (2002), in which a population of the weed Polygonum persicaria is subject to a two-year rotation of either carrots $(\mathrm{C})$ or wheat $(\mathrm{W})$. The population is structured by the depth at which seeds occur, as depth affects the probabilities of seedling emergence, seed survival, and movement to other layers in the soil by cultivation. In this case, there are four soil layers, each 5 $\mathrm{cm}$ deep. The population is projected from one spring to the following spring, just after the crop has been sown, so the population is formed only of seeds. As seeds are produced by plants, each matrix element summarizes information on seedling emergence from each layer $\left(\alpha_{l}\right)$, survival of plants emerged from layer $l\left(v_{l}\right)$, seed production of plants emerged from layer $l\left(\gamma_{l}\right)$, movement of seeds from layer $l$ to layer $k\left(\delta_{k l}\right)$, mortality of seeds at each soil depth before plowing $\left(\mu_{l}\right)$, and survival of seeds at each soil depth after plowing $\left(\sigma_{k}\right)$ :

$$
p_{k l}^{(h)}=\alpha_{l} v_{l} \gamma_{l} \delta_{k l} \sigma_{k}+\left(1-\alpha_{l}\right)\left(1-\mu_{l}\right) \delta_{k l} \sigma_{k}
$$

where $p_{k l}^{(h)}$ is the $k l$ th element of a matrix $\mathbf{P}$ for the $h$ th crop (see Eq. 2), and all parameter values correspond to those found in the crop occurring in the $h$ th phase.
Each crop and its associated management have different effects on demographic transitions, such as seedling emergence, plant survival, and fecundity (Table 2). For example, seedling emergence differs between the crops due to differences in sowing time, while plant survival differs due to differences in the efficacy of mechanical control in each crop. Another difference is the type of soil cultivation that is applied after each crop has been harvested. In the carrot crop, a rigid-tine implement is used which does not invert the soil, so seeds stay more or less where they are. After the wheat crop has been harvested, a plow is used that inverts the soil and consequently many seeds from the top layer are moved to deeper soil layers.

When the value of the transition elements are calculated using (Eq. 20) and the parameter values in Table 2, very different transition matrices are obtained for each crop (Table 3). In the carrot matrix $\mathbf{C}$, the highest transitions lie mainly along the diagonal, while in the wheat matrix $\mathbf{W}$, the highest values are found for the elements projecting seeds from the top layer to deeper layers. We use these two matrices to study the cyclic permutations of the simplest environmental sequence: the two-year cycles carrots-wheat $(\mathrm{CW})$ and wheat-carrots (WC). For heuristic purposes and the desire for simplicity, we focus on the composite matrix elements rather than the underlying parameters and suppose that each transition element can be perturbed independently.

The long-term population dynamics for the cyclic permutations $\mathrm{CW}$ and $\mathrm{WC}$ are identical in all but their phase (Fig. 1). Consequently, the long-term population growth rates over one cycle are the same for these two permutations, at 1.01 per two-year cycle (the population growth rates when $\mathrm{C}$ or $\mathrm{W}$ are grown singly are, respectively, 0.762 and 1.821 per year). The long-term periodic elasticities (Table 3 ) are also independent of the cyclic permutation of the environments (Caswell 2001). 
TABLE 3. Life-history transition matrices for the weed species Polygonum persicaria growing in carrot (C) and wheat (W) environments, and the elasticity values and stable depth structure associated with each crop and resulting from asymptotic analysis of the cyclic permutations $\mathrm{CW}$ and WC.

\begin{tabular}{|c|c|c|c|c|c|c|c|c|c|}
\hline \multirow[b]{2}{*}{ Phase and class } & \multicolumn{4}{|c|}{ Transition matrices } & \multicolumn{4}{|c|}{ Elasticity matrices (cycles $\mathrm{CW}$ and WC) } & \multirow[b]{2}{*}{ Stable structure } \\
\hline & 1 & 2 & 3 & 4 & 1 & 2 & 3 & 4 & \\
\hline \multicolumn{10}{|l|}{ Carrots } \\
\hline 1 & 0.126 & 0.231 & 0.016 & 0 & 0.160 & 0.265 & 0.036 & 0 & 0.086 \\
\hline 2 & 0.041 & 0.350 & 0.120 & 0 & 0.006 & 0.040 & 0.034 & 0 & 0.136 \\
\hline 3 & 0.011 & 0.105 & 0.544 & 0.128 & 0.002 & 0.015 & 0.157 & 0.051 & 0.331 \\
\hline 4 & 0.004 & 0.007 & 0.128 & 0.672 & 0 & 0.001 & 0.027 & 0.196 & 0.448 \\
\hline \multicolumn{10}{|l|}{ Wheat } \\
\hline 1 & 0.176 & 0.168 & 0.296 & 0.232 & 0.011 & 0.016 & 0.069 & 0.073 & 0.162 \\
\hline 2 & 0.966 & 0.216 & 0.208 & 0.080 & 0.127 & 0.045 & 0.105 & 0.055 & 0.147 \\
\hline 3 & 3.512 & 0.240 & 0.160 & 0.096 & 0.178 & 0.019 & 0.031 & 0.025 & 0.292 \\
\hline 4 & 4.039 & 0.168 & 0.144 & 0.384 & 0.146 & 0.010 & 0.020 & 0.072 & 0.399 \\
\hline
\end{tabular}

Notes: In the matrices, the element at row 1, column 2 quantifies the transition from class 2 to class 1 . The values of the transition elements are derived from Eq. 20, and the values are in Table 2. The asymptotic growth rate resulting from permutations CW and WC is $\lambda_{1}=1.01$ per two-year cycle.

In the case of the permutations $\mathrm{CW}$ and $\mathrm{WC}$, perturbations to $c_{11}, c_{12}, c_{33}$, and $c_{44}$ of the carrot phase will have the largest effect, while perturbations to elements $w_{21}, w_{31}$, and $w_{41}$ of the wheat phase are most important (Table 3). Likewise, the average long-term stable structure is identical for all cyclic permutations of the environments, but the stage structure does change within a cycle. After a $\mathrm{C}$ year, only about $9 \%$ of the seeds are in the upper soil layer, while after a $\mathrm{W}$ year, more than $15 \%$ are in the upper soil layer (Table 3).

We first examine how cyclic permutations affect transient population size and the pattern of transient elasticities. We consider cyclic permutations $\mathrm{CW}$ and WC, and use a total initial population size of $n(0)=100$, with an initial structure where $70 \%$ of the seeds are concentrated in the upper layer (class 1) and the

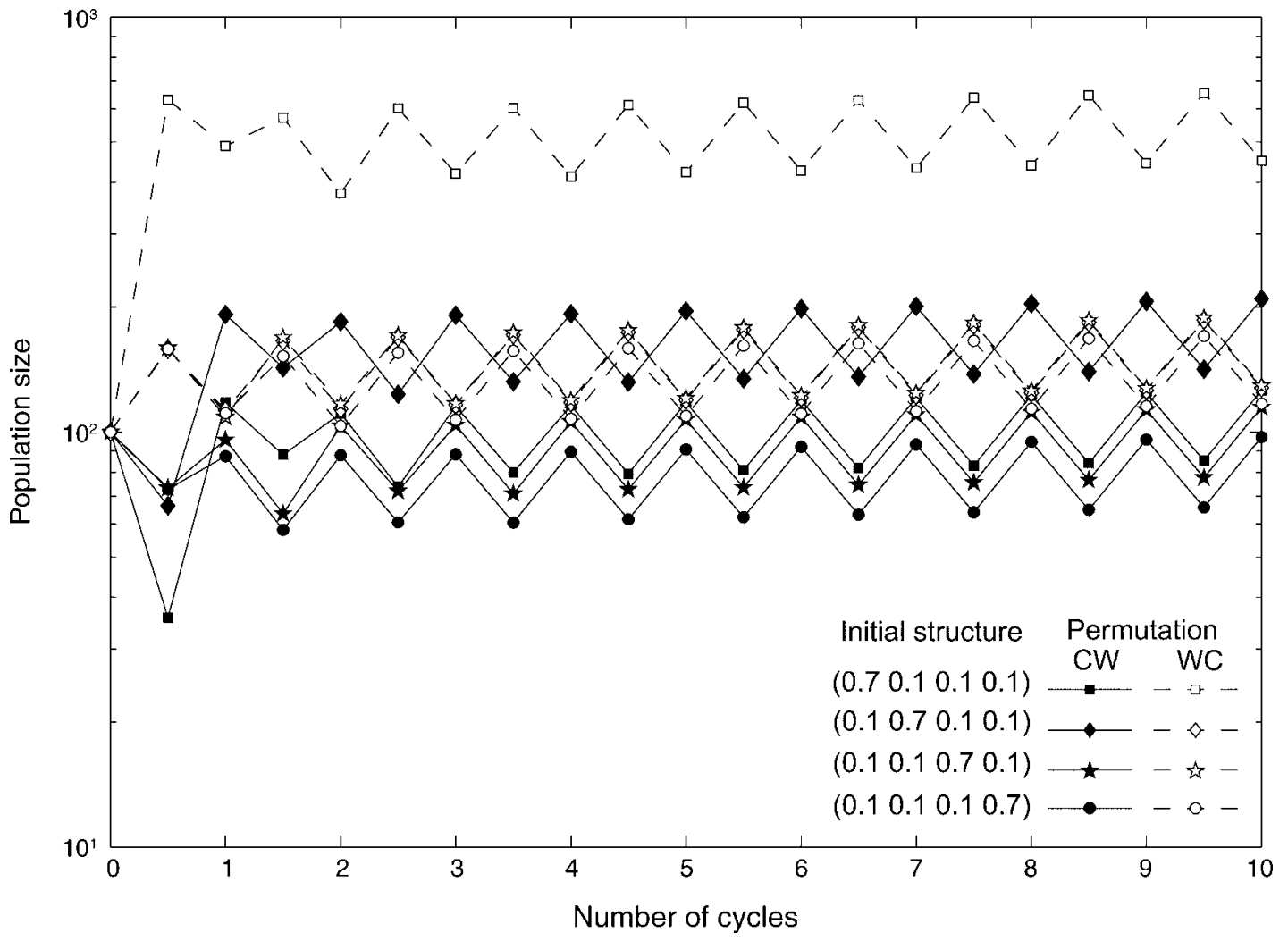

FIG. 1. The effects of initial population structure and cyclic permutation on population size at the end of each phase of a twophase cycle (carrots, C; wheat, W). Each line shows the population size for a different combination of initial population structure and cyclic permutation. The total initial population size is $N(0)=100$. 

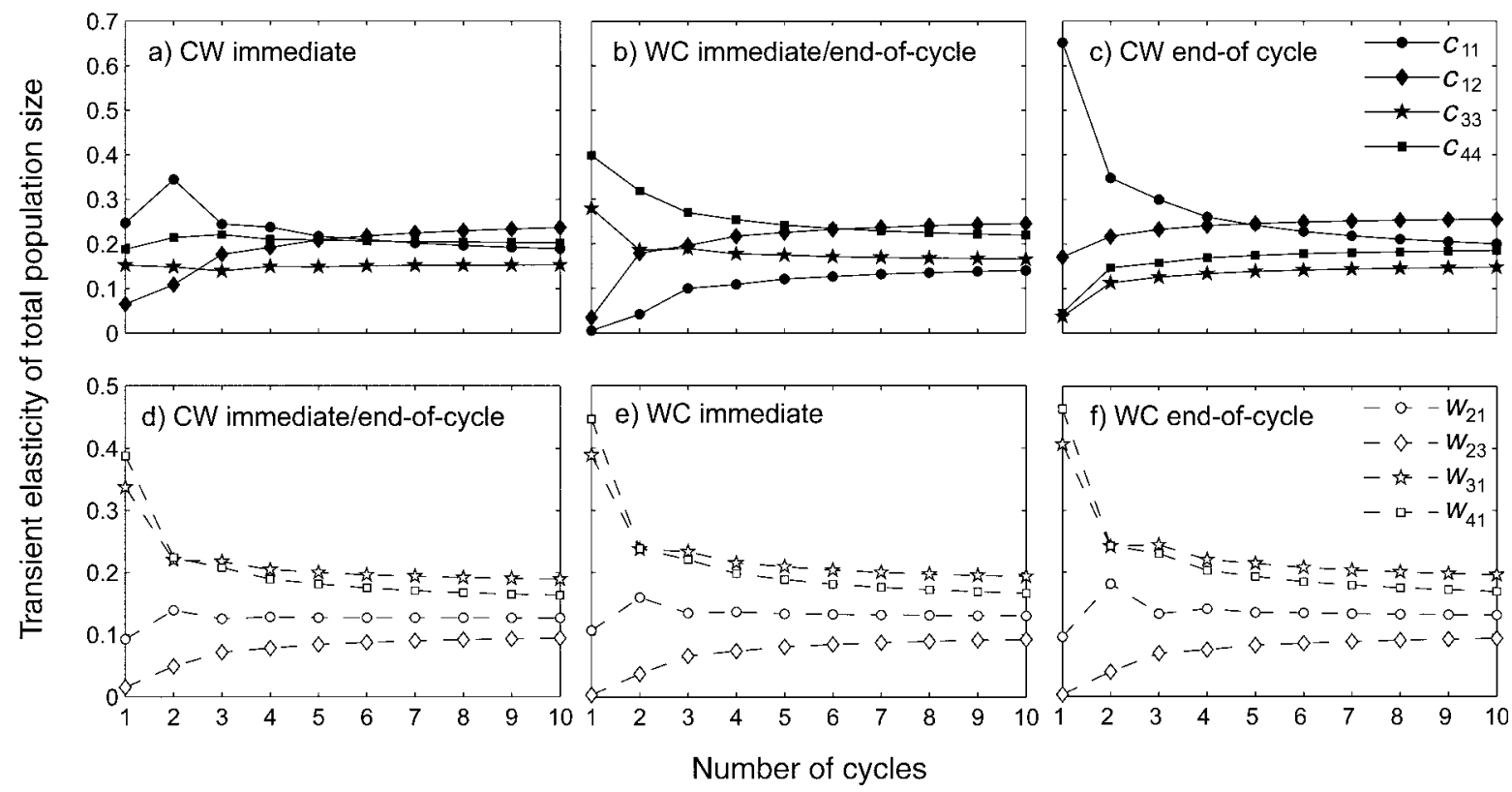

FIG. 2. Immediate and end-of-cycle transient elasticities of population size, scaled for time, for the two cyclic permutations CW and WC. The total initial population has 100 seeds, and the initial population vector is $n(0)=(70,10,10,10)^{\top}$. Results are shown only for elements to which the transient elasticities are about 0.1 or greater at some time during the first 10 cycles. Panels (a)-(c) give the elasticities for the carrot phase, and panels (d)-(f) give the elasticities for the wheat phase. When the last phase of a permutation is perturbed, then the immediate and end-of-cycle elasticities are the same.

remaining $30 \%$ are distributed evenly among the remaining layers. We then examine how different initial conditions interact with cyclic permutations. We again use a total initial population size of $n(0)=100$ and systematically consider four initial stage distributions such that, in each set of initial conditions, $70 \%$ of the seeds are concentrated in a different layer and the remaining $30 \%$ are evenly divided among the remaining three layers. Finally, by contrasting immediate and endof-cycle elasticities, we examine how elasticities of population size are affected by the time of observation. We present our results in terms of time-scaled elasticities (Fox and Gurevitch 2000), which can be compared with the elasticities of the long-term population growth rate. To simplify interpretation, we focus on elements that have an elasticity of population size greater than 0.1 at some point in time.

\section{Cyclic permutations of the environments}

The cyclic permutations carrots-wheat $(\mathrm{CW})$ and wheat-carrots (WC) create different transient population dynamics which ultimately produce a constant relative difference in population size between the two cyclic permutations (Fig. 1). When the population is initially concentrated in the uppermost soil layer (class 1), the $\mathrm{CW}$ permutation has a population size that is nearly an order of magnitude lower than in the WC permutation (Fig. 1). These differences are mainly due to interactions between the initial environment and the initial population structure. When the population is initially concentrated in the uppermost layer (class 1), starting with a $\mathrm{C}$ phase reduces the population much more than starting with the $\mathrm{W}$ environment, because all the transition rates from class 1 are weaker in the $\mathrm{C}$ environment compared to the $\mathrm{W}$ environment (Table 3 ). In short, the population suffers an initial blow, from which it does not recover.

To make comparisons of the elasticities associated with transitions for a particular environment in different cyclic permutations (e.g., the elasticities of perturbations to elements of $\mathbf{C}$ in the cycles $\mathrm{WC}$ and $\mathrm{CW}$ ), we examine the response of a population immediately after a perturbation (i.e., immediate elasticities [Eq. 17a]), rather than waiting until the end of a cycle. If we were to use the elasticities at the end of each cycle (Eq. 14), then perturbations at different phases would have different following environments, confounding the interpretation of differences (compare Fig. 2a and c).

The order of the environments affects both the pattern of elasticities and the persistence of the transient period (Fig. 2). The immediate elasticities for both the $\mathrm{C}$ and $\mathrm{W}$ environments differ between cyclic permutations (compare Fig. 2a with $2 \mathrm{~b}$ and Fig. $2 \mathrm{~d}$ with $2 \mathrm{e}$ ). With regard to the $\mathrm{C}$ environment, the pattern of elasticities is substantially different between the two permutations, as well as the persistence of the transients. In the $\mathrm{CW}$ cycle, the immediate elasticity associated with $c_{11}$ begins at 0.25 , increases to 0.35 and then decreases to its asymptotic value of 0.16 after more than 10 cycles (Fig. 2a). In contrast, in the WC cycle, the immediate elasticity associated with $c_{11}$ begins close to zero, increases to 0.1 by the third cycle, and then increases more slowly to reach its asymptotic value (Fig. 2b). The elasticities associated 
with elements $c_{33}$ and $c_{44}$ remain fairly close to their asymptotic values in the $\mathrm{CW}$ cycle, whereas in the WC permutation, they both start well above their asymptotic values and then gradually decline (Fig. 2b). In the $\mathrm{W}$ environment, the major difference in the immediate elasticities is that, in the WC permutation, the elasticities associated with elements $w_{31}$ and $w_{41}$ begin noticeably higher than in the CW permutation (Fig. 2d and 2e).

The different elasticity patterns can be understood by considering the initial population structure and its subsequent evolution due to application of the transition matrices. For example consider the initial conditions $n(0)=(70,10,10,10)^{T}$ and the permutation CW. Recall that in the $\mathrm{C}$ environment, the largest transition from the uppermost soil layer (class 1) is that of remaining in the top layer $\left(c_{11}=0.126\right.$, Table 3$)$ and all transitions of $\mathrm{C}$ lead to a decrease in the seed bank population. In the $\mathrm{W}$ environment, however, elements $w_{31}$ and $w_{41}$, which move seeds out of class 1, lead to large increases in the population. Consequently, any actions that lead to an increase in the population of the uppermost soil layer during the first carrot phase will be magnified by the end of the succeeding wheat crop. As the population is initially concentrated in the uppermost soil layer, rather than the second soil layer (class 2), the elasticity associated with element $c_{11}$ has the highest value $\left(e_{11}(1)=0.25\right.$, Fig. 2). The increase in the immediate elasticity by the end of the second carrot crop $\left(e_{11}(2)=\right.$ 0.34 ) is due mainly to the continued effect of the increase in $c_{11}$ during the first cycle, as it leads to a large increase in the population during the wheat crop. This increase in population size is greater than that produced by any other perturbation and so the elasticity associated with $c_{11}$ is the largest in the carrot phase in the second cycle. A perturbation of $c_{11}$ in the second cycle, would not, however, lead to a larger change in population size than a perturbation to $c_{12}$, because most of the population is concentrated in the second layer at the start of the second cycle. In other words, as the proportion of the population in the second layer increases at the end of each wheat crop, the elasticity associated with $c_{11}$ decreases and that associated with $c_{12}$ increases. A similar line of reasoning can be used to explain the pattern of elasticities associated with other elements and permutations.

\section{Interaction of initial conditions and cyclic permutations}

Even for this simple, four-stage model, the initial population structure has a long-term effect on the total population size of nearly an order of magnitude (Fig. 1). In the WC cycle, relatively large population sizes result from an initial structure that concentrates seeds in the uppermost soil layer (class 1). However, in the $\mathrm{CW}$ permutations, a larger population is achieved when the population is concentrated in the second layer (class 2), and even results in a population that is higher than that for several of the WC populations. These differences emerge from differences in the transient dynamics in the first couple of cycles due to interaction of the initial conditions with the cyclic permutation. After the transient period, all populations approach the same long-term growth rate. The effect of initial stage structure on the population size can be analyzed by looking at the sensitivity of the population size to the initial stage structure (Fox and Gurevitch 2000).

The initial population structure can also have a large effect on the elasticities of population size. For the immediate elasticity associated with element $c_{44}$, all combinations of initial conditions with cyclic permutations result in different approaches to the asymptotic value (Fig. 3). In the $\mathrm{CW}$ cycle, when the initial population is concentrated in class 1 , the immediate elasticity of population size to perturbations of $c_{44}$ quickly reaches its asymptotic value. In contrast, when the initial population is concentrated in the deepest soil layer (class 4 ), the elasticity begins at 0.65 and slowly decreases to reach its asymptotic value of 0.196 (Table $3)$. In the WC rotations, when the population is concentrated in either the top or bottom layer (class 1 or 4), $e_{44}$ begins higher than if the population is concentrated in either of the middle two layers (class 2 or 3). Even when the population is at its stable structure, the transient elasticities are still quite far from their asymptotic values (Fig. 3). There is, however, no difference between cyclic permutations (see Discussion for an explanation of this result).

\section{Effects of the time of observation}

Another aspect to consider is the time of observation relative to phases in the environmental cycle. The impact of a perturbation will differ depending on whether one observes the population directly after the perturbation, or at some other point in the cycle. For example, with regard to perturbations of $c_{11}$ and $c_{12}$ in the $\mathrm{CW}$ cycle and having the initial population concentrated in the uppermost soil layer (class 1), perturbations will have a larger effect when observed at the end of the entire $\mathrm{CW}$ cycle, rather than if the population is observed directly after the $\mathrm{C}$ cycle (Fig. 2a and c).

\section{Discussion}

Previous methods for examining transient dynamics of matrix population models have been limited to nonperiodic systems (Fox and Gurevitch 2000, Yearsley 2004). We have presented new analytic methods for examining the transient sensitivities and elasticities of periodic matrix models, and have demonstrated how they can be applied. An important outcome of our work is a method for calculating the effects of perturbations immediately after a particular environmental phase, rather than just at the end of a cycle of environments. This method enables comparison of the effect of perturbations to life history-transitions, between environments in different cyclic and noncyclic permutations. Below, we discuss implications for population management in periodic systems, and discuss insights from our 


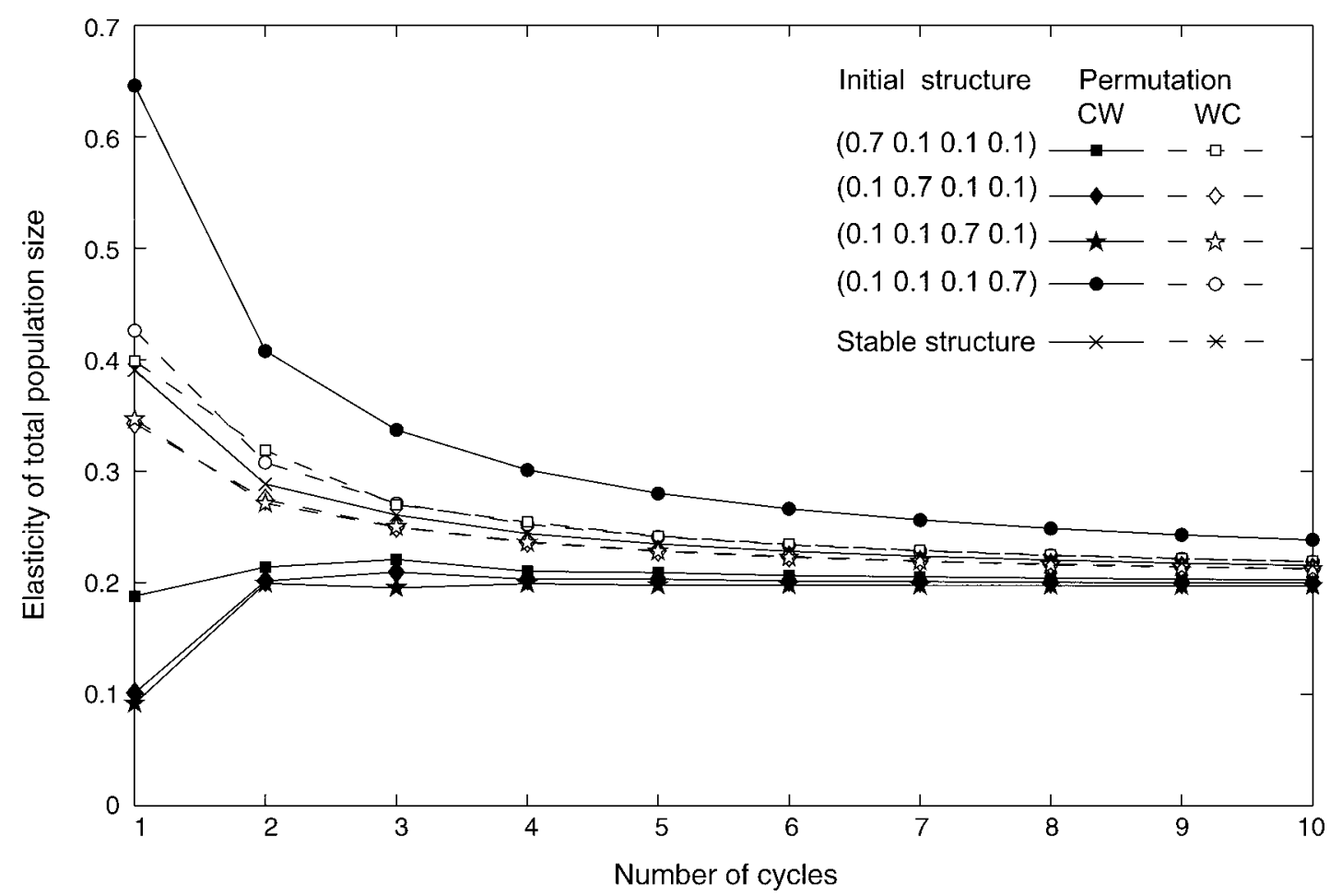

FIG. 3. The effect of different initial stage structures on the immediate elasticity (scaled by time) of population size to perturbations to the transition for remaining in the bottom layer in the carrot phase $\left(c_{44}\right)$, for both permutations (CW and WC). The asymptotic elasticity associated with $c_{44}$ is $e_{44}=0.196$ (Table 3 ).

analysis that are also applicable to non-periodic matrix models.

\section{Understanding and managing periodic systems}

Our methods can be used to answer several questions relevant to the management and monitoring of cycles regardless of whether they are composed of independent or dependent environments. One question concerns when, in an environmental cycle, to make a major disturbance to the population vector, such as restocking fisheries or reducing the population size of an invasive species. As we have shown, the effect of a major disturbance to the population vector will depend on when in the cycle it is carried out. A second question is how to design effective monitoring programs. The fact that the effect of a perturbation depends on when it is observed in the cycle allows managers, for example, to target monitoring at the points in the cycle when the effect is expected to be greatest and therefore most discernible.

Another question is which cyclic permutation to use when implementing a new management strategy. This question is relevant for systems composed wholly or partly of independent environments. One criterion for choosing a permutation is target population size. Given the initial population size, a manager could implement the cyclic permutation that achieves the target population size the fastest. Alternatively, a manager may wish to take advantage of a particular pattern of elasticity values, as some transitions may be more practical to perturb. The pattern of elasticities becomes particularly relevant when the difference in population size between cyclic permutations is relatively small and so the criteria of target population size is not an issue. The cyclic permutation, therefore, could be chosen such that perturbations to those easily perturbed transitions will have the largest impact. However, because an observed effect is due to past as well as the most recent perturbation, careful analysis of the interaction of initial conditions with the life-history transitions is imperative. Finally, when there is uncertainty concerning initial population structure, a manager could choose to use the cyclic permutation that is least sensitive to the initial population structure, e.g., in our example, using permutation WC rather than $\mathrm{CW}$, as shown in Fig. 3.

\section{Remarks on perturbation analysis of matrix population models}

As transient sensitivity analysis of matrix population models has only recently received scrutiny, there are some aspects concerning perturbation analysis of both periodic and non-periodic matrix models that merit highlighting. These aspects are related to the interpretation of transient elasticities and sensitivities and transient dynamics even when the population is in the stable distribution. 
First, the perturbation analysis shown in this study corresponds to a permanent change in a life-history transition rate (i.e., a press perturbation sensu Bender et al. [1984]). For example, the high elasticity associated with element $c_{11}$ in the second cycle of the carrot-wheat permutation (Fig. 2a) is due to the perturbation during the first cycle, and its interaction with the first wheat crop. Consequently one cannot choose to perturb a transition when the elasticity is the highest and expect the outcome to be similar to the results of the analysis. Furthermore, one cannot simply perturb a single element for several time steps and then change to another element and expect similar results as given by the transient elasticity analysis. Rather, a new transient analysis must be carried out using the population size and structure resulting from the first perturbation events.

Second, even if the population is at its stable stage structure, it will not necessarily settle into the asymptotic elasticities immediately (see example in Yearsely [2004] and Fig. 3). In fact, it can take many generations until the ranking of the asymptotic results is achieved, by which time the system may have been disturbed by unforeseen factors. This is a consequence of the fact that a perturbation will also perturb the population structure and can be seen by examining the first component $\left(\left(\mathbf{S}\left(t ; a_{k l}\right)\left\{[n(0)] / \| \mathbf{w}^{(1)}||\right\}\right)\right)$ of Eq. 3, because $\Delta \mathbf{n}(0)=0$ (Yearsley 2004). With regard to periodic systems, there may be slight differences in the immediate elasticities between cyclic permutations depending on which stable structure one examines, but one would expect the differences to be negligible (Fig. 3).

\section{Further application of our methods}

To focus attention on aspects of transient analysis that are particular to periodic systems, we considered transient dynamics of total population size only. In many cases, this may be sufficient, as managers may be more interested in maximizing or minimizing total population size rather than that of a single class. Furthermore, it appears that the pattern of transient sensitivities and elasticities of population size tend to be dominated by a single stage (S. K. Mertens, J. M. Yearsley, F. van den Bosch, and C. A. Gilligan, unpublished results). Consequently, while a particular stage may be very sensitive to a given transition, its impact on total population size may be relatively small. Using our methods, all of the stage specific analyses illustrated by Fox and Gurevitch (2000) and Yearsley (2004) can be carried out. These can be useful for comparing predictions with observations when some population classes are difficult to observe.

Our analysis has focused on perturbing a single lifehistory transition, while other transitions are kept constant. This approach is useful for highlighting important transitions. However, because the essence of transient elasticities and sensitivities is that they change with time, in a management context it can be particularly relevant to explore the effects of changing more than one element at a time. As one element becomes more important, another element will decrease in importance, therefore it may be useful to consider perturbations to multiple elements. Caswell (2001) shows how to calculate multiple perturbations; these can easily be extended to transient analysis and periodic systems with the methods described here and by Fox and Gurevitch (2000) and Yearsley (2004).

\section{ACKNOWLedgMents}

Rothamsted Research is supported by the Biotechnology and Biological Sciences Research Council (BBSRC). This project was supported by BBSRC grant D17567. J. Yearsley was supported by the Scottish Executive through the FIFEI project. C. A. Gilligan was supported by a BBSRC Professorial Fellowship.

\section{Literature Cited}

Beissinger, S. R. 1995. Modeling extinction in periodic environments: Everglades water levels and snail kite population viability. Ecological Applications 5:618-631.

Bender, E. A., T. J. Case, and M. E. Gilpin. 1984. Perturbation experiments in community ecology: theory and practice. Ecology 65:1-13.

Benton, T., and A. Grant. 1999. Elasticity analysis as an important tool in evolutionary and population ecology. Trends in Ecology and Evolution 14:467-471.

Caswell, H. 2001. Matrix population models: construction, analysis, and interpretation. Second edition. Sinauer Associates, Sunderland, Massachusetts, USA.

Caswell, H., and M. C. Trevisan. 1994. Sensitivity analysis of periodic matrix models. Ecology 75:1299-1303.

Cousens, R., and S. R. Moss. 1990. A model of the effects of cultivation on the vertical distribution of weed seeds within the soil. Weed Research 30:61-70.

de Kroon, H., J. van Groenendael, and J. Ehrlen. 2000. Elasticities: a review of methods and model limitations. Ecology 81:607-618.

Elhay, S., G. M. L. Gladwell, G. Golub, and Y. M. Ram. 1999. On some eigenvector-eigenvalue relations. SIAM Journal of Matrix Analysis and Applications 20:563-574.

Endress, B. A., D. L. Gorchov, and R. B. Noble. 2004. Nontimber forest product extraction: effects of harvest and browsing on an understory palm. Ecological Appplications 14:1139-1153.

Escalante, S., C. Montana, and R. Orellana. 2004. Demography and potential extractive use of the liana palm, Desmoncus orthacanthos Martius (Arecaceae), in southern Quintana Roo, Mexico. Forest Ecology and Management 187:3-18.

Fox, G. A., and J. Gurevitch. 2000. Population numbers count: tools for near-term demographic analysis. American Naturalist 156:242-256.

Gross, K., J. R. Lockwood, C. C. Frost, and M. F. Morris. 1998. Modeling controlled burning and trampling reduction for conservation of Hudsonia montana. Conservation Biology 12:1291-1301.

Hoffmann, W. A. 1999. Fire and population dynamics of woody plants in a neotropical savanna: matrix model projections. Ecology 80:1354-1369.

Keyfitz, N. 1971. On the momentum of population growth. Demography 8:71-80.

Koons, D. N., J. B. Grand, B. Zinner, and R. F. Rockwell. 2005. Transient population dynamics: relations to life history and initial population state. Ecological Modelling 185:283297.

Lefkovitch, L. P. 1971. Some comments on the invariants of population growth. Pages 337-360 in G. P. Patil, E. C. 
Pielou, and W. E. Water, editors. Statistical ecology. Volume 2. Pennsylvania State University Press, University Park, Pennsylvania, USA.

Lesnoff, M. 1999. Dynamics of a sheep population in a Sahelian area (Ndiagne district in Senegal): a periodic matrix model. Agricultural Systems 61:207-221.

Lesnoff, M., P. Ezanno, and H. Caswell. 2003. Sensitivity analysis in periodic matrix models: a postscript to Caswell and Trevisan. Mathematical and Computer Modelling 37: 945-948.

Liebman, M., and E. Dyck. 1993. Crop rotation and intercropping strategies for weed management. Ecological Applications 3:92-122.

May, M. J., G. T. Champion, A. M. Dewar, A. Qi, and J. D. Pidgeon. 2005. Management of genetically modified herbicide-tolerant sugar beet for spring and autumn environmental benefit. Proceedings of the Royal Society of London B 272:111-119.

Mertens, S. K., F. van den Bosch, and J. A. P. Heesterbeek. 2002. Weed populations and crop rotations: exploring dynamics of a structured periodic system. Ecological Applications 12:1125-1141.
Press, W. H., S. A. Teukolsky, W. T. Vetterling, and B. P. Flannery. 1996. Numerical recipes in C. Cambridge University Press, Cambridge, UK.

Satterthwaite, W. H., E. S. Menges, and P. F. QuintanaAscencio. 2002. Assessing scrub buckwheat population viability in relation to fire using multiple modeling techniques. Ecological Applications 12:1672-1687.

Shea, K., H. P. Possingham, W. W. Murdoch, and R. Roush. 2002. Active adaptive management in insect pest and weed control: intervention with a plan for learning. Ecological Applications 12:927-936.

Silva, J. F., J. Raventos, H. Caswell, and M. C. Trevisan. 1991. Population responses to fire in a tropical savanna grass, Andropogon semiberbis: a matrix model approach. Journal of Ecology 79:345-356.

Strang, G. 1980. Linear algebra and its applications. Second edition. Academic Press, Orlando, Florida, USA.

Walters, C. 1986. Adaptive management of renewable resources. Macmillan, New York, New York, USA.

Yearsley, J. M. 2004. Transient population dynamics and shortterm sensitivity analysis of matrix population models. Ecological Modelling 177:245-258.

\section{APPENDIX A}

A discussion of the effects of cyclic permutations and initial conditions on the transient dynamics of a tropical grass (Andropogon semiberbis) exposed to alternating burn and nonburn environments (Ecological Archives E087-141-A1).

\section{APPENDIX B}

Derivation of the equation for within-cycle sensitivity (Ecological Archives E087-141-A2).

\section{SUPPLEMENT}

Matlab scripts for calculating the sensitivity and elasticity of population size for periodic matrix models (Ecological Archives E087-141-S1). 